\title{
Design and Fabrication of a Three-Dimensional In Vitro Model of Vascular Stenosis
}

Rebecca S Jones ${ }^{1}$, Pin Hsuan Chang ${ }^{1,2}$, Tzlil Perahia ${ }^{3}$, Katrina A Harmon ${ }^{3}$, Lorain Junor ${ }^{3}$, Michael J Yost $^{4}$, Daping Fan ${ }^{1,3}$, John F Eberth ${ }^{1,3}$, and Richard L Goodwin ${ }^{5}$

${ }^{1 .}$ Biomedical Engineering Program, University of South Carolina, Columbia, SC, USA

2. Department of Civil and Environmental Engineering, University of Michigan, Ann Arbor, MI, USA

3. Department of Cell Biology and Anatomy, School of Medicine, University of South Carolina, Columbia, SC, USA

4. Department of Surgery, Medical University of South Carolina, Charleston, SC, USA

5. Department of Biomedical Sciences, School of Medicine, University of South Carolina, Greenville, SC, USA

Vascular stenosis, a common condition broadly characterized as abnormal blood vessel narrowing, arises from defective developmental processes or adult pathologies often associated with atherosclerosis and results in increased resistance to blood flow that can progress to partial or complete occlusion of the vessel with numerous fatal outcomes. Despite the wide variety in etiology of this vascular pathology, a common series of sequelae involving each of the three representative vascular cell types occurs that is characterized by endothelial cell (EC) activation, excessive smooth muscle cell (SMC) proliferation, and extracellular matrix (ECM) remodeling via fibroblasts (FBs). Without intervention, this pathology will progress, leading to critical stenosis, occlusion, and, potentially, vessel rupture, however the cellular interactions and hemodynamic mechanisms that regulate this progression are not well understood [1]. From our prior work with fabricating 3D models of cardiovascular tissues to investigate hemodynamicdriven remodeling in the development of several tissues, it is apparent that mechanical loading plays a pivotal role in the behavior of cells embedded within the vascular walls or on the surface of these matrices, yet the molecular mechanisms that regulate the mechanotransduction pathways involved in the initiation and the progression of vascular pathology have yet to be determined [2-5]. By fabricating tubular cellularized collagen arterial scaffolds with a stenotic morphology (cytotubes) and culturing them with a custom dynamic fluid-flow bioreactor in the appropriate 3D physical environment, these pathological conditions can be studied in an in vitro setting, thereby elucidating novel mechanistic processes not previously substantiated with conventional culture models. Thus, the purpose of this study was to generate an in vitro, 3D arterial model capable of supporting the growth and development of an array of characteristic vascular cells in an environment that can provide dynamic mechanical stimuli.

In the present study, the cytotubes were produced using a type I collagen casting system to generate an arterial model with stenotic morphology containing various combinations of vascular cells in the tube wall or lumen. For static culture in petri dishes, cytotubes were fabricated with the following cell combinations: FB-only (wall), SMC-only (wall), EC-only (lumen), and SMC-EC (wall and lumen, respectively). For static (no-flow) and dynamic (flow at $0.2 \mathrm{~mL} / \mathrm{min}$ ) culturing in bioreactors, cytotubes contained FBs and bone marrow primary cells (BMPCs) within the tube wall. Cell viability, distribution, morphology, and tube length and diameter contraction were examined using confocal microscopy and ANOVA analysis. We found that all cell types remained viable during culture and developed locationand time-dependent morphologies, as seen in Figure 1 with EC-only cytotubes. Analysis of cellularized collagen tube diameter and length contraction found that constructs containing SMC-EC co-cultures contracted more than EC-only constructs but less than SMC-only cytotubes, indicating that heterotypic, cell-cell interactions play an important role during histogenesis (Figure 2). The FB-BMPC cytotubes 
were cultured on bioreactors with and without applied flow for 2 days, resulting in increased cellular alignment at the edges of the wall and interconnected cellular networks in cytotubes cultured with flow (Figure 3). Our fabrication approach has allowed us to create arterial scaffolds containing several combinations of characteristic vascular cells that can be cultured with our bioreactor under static or dynamic flow conditions. These results support the premise that this collagen fabrication technique is capable of producing cellularized constructs with multiple vascular cell types and specific 3D geometries that can be used in combination with our novel fluid-flow bioreactor to further investigate the cellular and mechanical mechanisms of vascular disease in a dynamic in vitro culturing system [6].

\section{References:}

[1] J-J Chiu and S Chien, Physiological reviews 911 (2011), p. 327.

[2] C Hahn and MA Schwartz, Nature reviews. Molecular cell biology 101 (2009), p. 53.

[3] H Tan et al, Developmental biology 3742 (2013), p. 345.

[4] SV Biechler et al, Frontiers in physiology 5 (2014), p. 225.

[5] RL Goodwin et al, Microscopy and Microanalysis 21 S3 (2015), p. 619.

[6] The authors acknowledge funding from the National Institutes of Health $(\mathrm{NIH})$ with the R01 grant HL-116626 to DF. Dr. Robert L. Price is thanked for his helpful guidance and critique of this work.

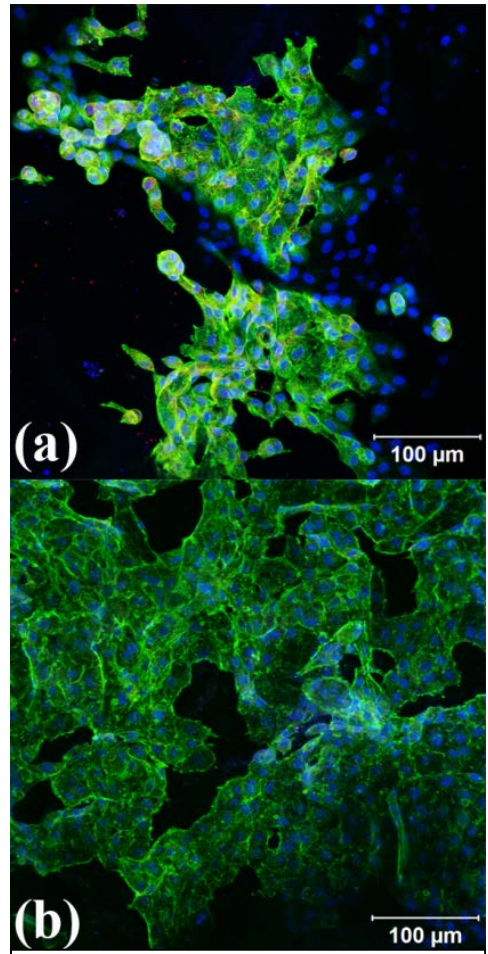

Figure 1. Day 1 (a) and Day 7 (b) cytotubes with lumen-seeded ECs showed increasing cell confluency and cortical arrangements of f-actin (green). Blue staining is DAPI, and red is $\alpha \mathrm{SMA}$.
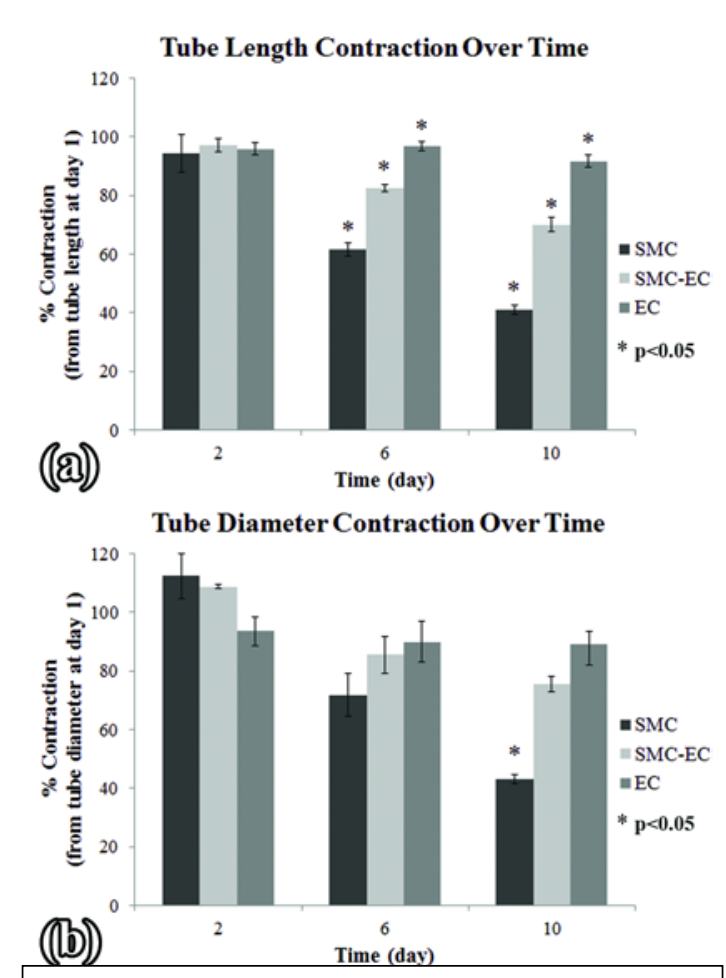

Figure 2. Cytotube length and diameter contraction varied with cell composition. Mean differences of contraction length were significant between all culture types for Days 6 and 10 (a). Mean differences of contraction diameter were only significant at Day 10 for SMC vs. SMCEC and SMC vs. EC (b).

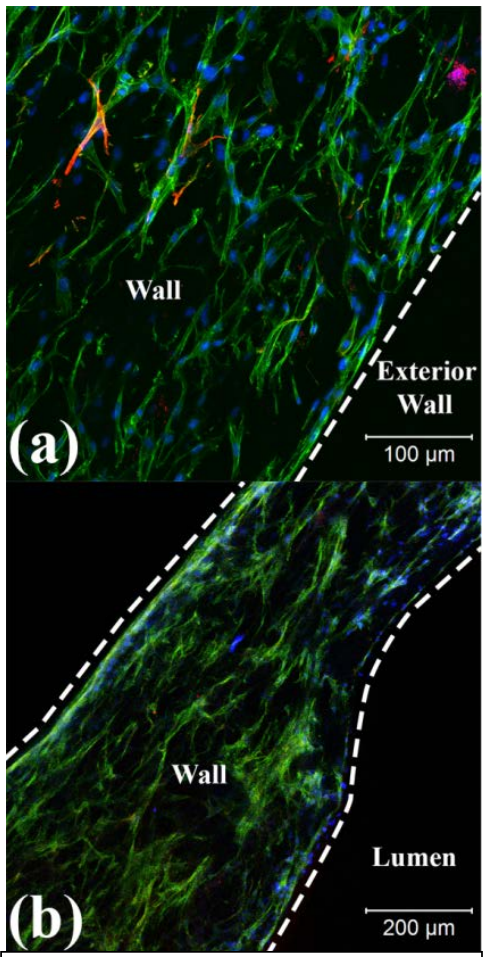

Figure 3. Day 2 FB-BMPC cytotubes with no flow (a) and a $0.2 \mathrm{~mL} / \mathrm{min}$ flow rate (b) had differences in $\mathrm{f}$ actin (green) networks and cell alignment at wall edges. Blue staining is DAPI. and red is $\alpha$ SMA. 